\title{
Point-of-care assessment of platelet aggregation in infants and children with cyanotic and acyanotic heart disease \\ undergoing open-heart surgery.
}

\section{SAINT-LUC}

Cliniques universitaires Saint Luc; Université Catholique de Louvain, Dept of Anaesthesiology, Brussels, Belgium

\section{Background and goal of the study}

Conflicting results exist regarding platelet (PL) count/function in infants with Cyanotic (Cyan) compared to Acyanotic (Acyan) heart disease ${ }^{1-2}$ We investigated this point and analysed the transfusion rate of PL concentrates in a homgeneous group of infants with these pathologies.

\section{Material and methods}

This is a subanalysis of a prospective double-blinded randomised trial (NCT02567786) evaluating the effectiveness of a pump prime based on FFP vs Plasmalyte ${ }^{\circledR}$ in infants weighing 7-15 kg and undergoing open-heart surgery. Blood was drawn for PL count and PL function evaluated by Point Of Care (POC) tests Multiplate ${ }^{\circledR}$ (ADP, ASPI, TRAP) and ROTEM ${ }^{\circledR}$ after the induction of anaesthesia (T1), at the end of cardiopulmonary bypass (T2) and at Paediatric Intensive Care Unit (PICU) arrival (T3). Transfusion was based on clinical bleeding and guided with POC tests. A repeated measure ANOVA was performed to analyse the effect of time and group on PL count and function. A Student's t-test and Fisher'exact were used to respectively compare continuous and categorical variables between both groups.

\section{Results and discussion}

After parental informed consent 30 children were included of whom 29 analysed (Table 1). As shown in Table 2 there was no significant difference between groups regarding POC tests and PL count. There was a statistically significant difference within groups for PL count, MCF EXTEM and ADP: $p<0.001$ T1 vs T2 and vs T3. This effect was much less observed for ASPI/TRAP. The transfusion of PL concentrates was similar between both groups.

Table 1: Baseline and perioperative data of patients

\begin{tabular}{|c|c|c|c|}
\hline & Cyan $(\mathrm{N}=14)$ & Acyan $(\mathrm{N}=15)$ & $\mathbf{p}$ \\
\hline Age (months) & $23 \pm 16$ & $25 \pm 16$ & 0.65 \\
\hline Weight (kg) & $10.4 \pm 2.5$ & $10.6 \pm 2.7$ & 0.83 \\
\hline $\begin{array}{c}\text { Baseline fibrinogen } \\
(\mathrm{mg} / \mathrm{dL})\end{array}$ & $300 \pm 86$ & $340 \pm 73$ & 0.20 \\
\hline Baseline $\mathrm{Hb}(\mathrm{mg} / \mathrm{dL})$ & $15.5 \pm 1.75$ & $11.5 \pm 1.67$ & $<0.001$ \\
\hline $\begin{array}{c}\text { Baseline PL count } \\
\left({ }^{*} 10^{3} / \mathrm{mm}^{3}\right)\end{array}$ & $336 \pm 103$ & $391 \pm 134$ & 0.23 \\
\hline $\begin{array}{c}\text { Baseline } \\
\text { Activated partial } \\
\text { thromboplastin time }\end{array}$ & $32 \pm 4$ & $31 \pm 4$ & 0.40 \\
\hline $\begin{array}{c}\text { Baseline } \\
\text { International } \\
\text { Normalized Ratio }\end{array}$ & $1.1 \pm 0.1$ & $1.1 \pm 0.1$ & 0.33 \\
\hline $\begin{array}{c}\text { Baseline } \\
\text { Prothrombin Time }\end{array}$ & $12.7 \pm 0.9$ & $12.3 \pm 1.2$ & 0.40 \\
\hline CPB time (min) & $169 \pm 67$ & $131 \pm 51$ & 0.11 \\
\hline $\begin{array}{c}\text { N patients } \\
\text { transfused } P L(O R)\end{array}$ & $4(29 \%)$ & $1(7 \%)$ & 0.17 \\
\hline $\begin{array}{l}\text { Transfusion PL } \\
(\mathrm{OR})^{\star}(\mathrm{mL} / \mathrm{kg})\end{array}$ & $0(0-8)$ & $0(0-0)$ & 0.13 \\
\hline $\begin{array}{c}\text { N patients } \\
\text { transfused PL (PICU) }\end{array}$ & $2(14 \%)$ & $1(7 \%)$ & 0.60 \\
\hline $\begin{array}{l}\text { Transfusion PL } \\
(\mathrm{PICU})^{\star}(\mathrm{mL} / \mathrm{kg})\end{array}$ & $0(0-0)$ & $0(0-0)$ & 0.56 \\
\hline $\begin{array}{c}\text { Blood loss } 6 \text { h post-op* } \\
(\mathrm{mL} / \mathrm{kg})\end{array}$ & $7.3(6.0-10.0)$ & $5.4(2.7-8.0)$ & 0.03 \\
\hline
\end{tabular}

Data are expressed as $\mathrm{N}(\%)$, mean $\pm \mathrm{SD}$ or median $(\mathrm{P} 25-\mathrm{P} 75)^{*}$
Table 2: PL count and POC tests

\begin{tabular}{|c|c|c|c|}
\hline & T1 & T2 & T3 \\
\hline 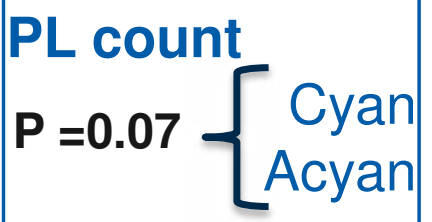 & $\begin{array}{l}336 \pm 103^{*} \\
391 \pm 134^{*}\end{array}$ & $\begin{array}{l}151 \pm 49 \\
179 \pm 98\end{array}$ & $\begin{array}{l}176 \pm 70 \\
205 \pm 81\end{array}$ \\
\hline $\begin{array}{l}\text { ADP AUC } \\
\mathbf{P}=0.22\end{array}\left\{\begin{array}{c}\text { Cyan } \\
\text { Acyan }\end{array}\right.$ & $\begin{array}{l}747 \pm 217^{*} \\
894 \pm 269^{*}\end{array}$ & $\begin{array}{l}288 \pm 202 \\
477 \pm 342\end{array}$ & $\begin{array}{l}378 \pm 206 \\
430 \pm 249\end{array}$ \\
\hline $\begin{array}{l}\text { ASPI AUC } \\
\mathbf{P}=\mathbf{0 . 2 7}\end{array}\left\{\begin{array}{c}\text { Cyan } \\
\text { Acyan }\end{array}\right.$ & $\begin{array}{l}1000 \pm 293^{* *} \\
1129 \pm 363^{* *}\end{array}$ & $\begin{array}{l}715 \pm 489 \\
976 \pm 727\end{array}$ & $\begin{array}{l}732 \pm 372 \\
775 \pm 498\end{array}$ \\
\hline $\begin{array}{l}\text { TRAP AUC } \\
\mathbf{P}=\mathbf{0 . 4 3}\end{array}\left\{\begin{array}{c}\text { Cyan } \\
\text { Acyan }\end{array}\right.$ & $\begin{array}{c}967 \pm 258^{\star *} \\
1165 \pm 303^{\star *}\end{array}$ & $\begin{array}{l}824 \pm 438 \\
914 \pm 727\end{array}$ & $\begin{array}{l}693 \pm 305 \\
656 \pm 372\end{array}$ \\
\hline $\begin{array}{l}\text { MCF EXTEM } \\
\mathbf{P}=\mathbf{0 . 1 6}\left\{\begin{array}{c}\text { Cyan } \\
\text { Acyan }\end{array}\right.\end{array}$ & $\begin{array}{l}61 \pm 5^{\star} \\
64 \pm 7^{*}\end{array}$ & $\begin{array}{c}48 \pm 8 \\
52 \pm 10\end{array}$ & $\begin{array}{l}50 \pm 8 \\
53 \pm 7\end{array}$ \\
\hline
\end{tabular}

${ }^{*} \mathrm{P}<0,001: \mathrm{T} 1$ vs $\mathrm{T} 2$ and $\mathrm{T} 1$ vs T3 ${ }^{\star *} \mathrm{P}<0,01: \mathrm{T} 1$ vs $\mathrm{T} 3$

ADP: Adenosine Diphosphate ASPI: Aspirine

TRAP: Thrombin Receptor Activating Peptide AUC: Area Under the Curve MCF: Maximum Clot Firmness

\section{Conclusions}

The results of this study show that PL number as well as PL function analysed by ROTEM ${ }^{B}$ significantly deteriorate after CPB in infants with Cyan as well as Acyan heart disease without significant difference between groups.

PL function test Multiplate ${ }^{\circledR}$ did not give additional information already available with PL count and ROTEM

The transfusion of PL concentrate was not significantly different between both groups although children with Cyan heart disease showed statistically significant more blood loss up to $6 \mathrm{~h}$ postoperatively. 